\title{
Student Perceptions of High-Value Care Education in Internal Medicine Clerkships
}

\author{
Amit Pahwa, MD ${ }^{1 *}$, Danelle Cayea, MD, MS², Amanda Bertram, MS ${ }^{1}$, Ariella Apfel, $\mathrm{MPH}^{1}$, Chad Miller, MD³, \\ Nick Van Wagoner, MD, $\mathrm{PhD}^{4}$, James Willig, MD, $\mathrm{MSPH}^{4}$, Reena Karani, MD, MHPE${ }^{5}$, Bimal Ashar, MD, MBA
}

\begin{abstract}
${ }^{1}$ Division of General Internal Medicine, The Johns Hopkins University School of Medicine, Baltimore, Maryland; ${ }^{2}$ Division of Geriatric Medicine and Gerontology, The Johns Hopkins University School of Medicine, Baltimore, Maryland; ${ }^{3}$ Department of Medicine, Tulane University School of Medicine, New Orleans, Louisiana; ${ }^{4}$ Division of Infectious Disease, University of Alabama School of Medicine, Birmingham, Alabama; ${ }^{5}$ Department of Medical Education, Icahn School of Medicine at Mount Sinai, New York, New York.
\end{abstract}

During internal medicine (IM) clerkships, course directors are responsible for ensuring that medical students attain basic competency in patient management through use of risk-benefit, cost-benefit, and evidence-based considerations. ${ }^{1}$ However, the students' primary teachers-IM residents and attendings - consistently role-model high-value care (HVC) perhaps only half the time. ${ }^{2}$ The inconsistency may have a few sources, including unawareness of the costs of tests and treatments ordered and little formal training in HVC. ${ }^{3-5}$ In addition, the environment at some academic institutions may reward learners for performing tests that may be unnecessary. ${ }^{6}$

We conducted a study to assess medical students' perceptions of unnecessary testing and the adequacy-inadequacy of HVC instruction, as well as their observations of behavior that may hinder the practice of HVC during the IM clerkship.

\section{METHODS}

When students completed their third-year IM clerkships at The Johns Hopkins University School of Medicine, the Icahn School of Medicine at Mount Sinai, the University of Alabama at Birmingham School of Medicine, and the Tulane University School of Medicine, we sent them a recruitment email asking them to complete an anonymous survey regarding their clerkship experiences with HVC. The clerkships' directors, who collaborated on survey development, searched the literature to quantify behavior thought to decrease the practice of HVC. The survey was tested several times with different learners and faculty to increase response process validity.

The SurveyMonkey online platform was used to administer the survey. Students were given 1 week after the end of their clerkship to complete the survey. Data were collected for the period October 2013 to December 2014. Each student was offered a $\$ 10$ gift certificate for survey completion. Each institution received exempt approval from its institutional review board.

\footnotetext{
*Address for correspondence and reprint requests: Amit Pahwa, MD, Division of General Internal Medicine, The Johns Hopkins University School of Medicine, 600 N Wolfe St, Nelson 215, Baltimore, MD 21287; Telephone: 410502-2128; Fax: 410-502-0923; E-mail: apahwa1@jhmi.edu
}

Received: May 5, 2016; Revised: August 19, 2016; Accepted: September 5, 2016 2017 Society of Hospital Medicine DOI 10.12788/jhm.2689
Survey respondents were divided into those who perceived HVC education as adequate and those who perceived it as inadequate. Chi-square tests were performed with Stata Version 12 (College Station, TX) to determine whether a student's perception of HVC education being adequate or inadequate was significantly associated with the other survey questions.

\section{RESULTS}

Of 577 eligible students, 307 (53\%) completed the survey. About $83 \%$ of the respondents reported noticing the ordering of laboratory or radiologic tests they considered unnecessary, and a majority $(81 \%)$ of those students noticed this activity at least once a week. Overall, $51 \%$ of the respondents thought their HVC education was inadequate. Significantly more of the students who perceived their HVC education as inadequate were uncomfortable bringing an unnecessary test to the attention of the ward team, rarely discussed costs, and rarely observed team members being praised for forgoing unnecessary tests (Table). Two significant associations were found: between institution attended and perceived adequacy-inadequacy of HVC education and between institution and frequency of cost discussions.

Most (78.5\%) students thought an HVC curriculum should be added to the IM clerkship, and 34.5\% thought the HVC curriculum should be incorporated into daily rounds. In regards to additions to the clerkship curriculum, most students wanted to round with phlebotomy (29\%) or discuss costs of testing on patients (26\%).

Students attributed the ordering of unnecessary tests and treatments to several factors: residents investigating "interesting diagnoses" (46\%), teams practicing defensive medicine $(43 \%)$, consultants making requests $(40 \%)$, attendings investigating "interesting diagnoses" $(27 \%)$, and patients making requests $(8 \%)$.

\section{DISCUSSION}

About $51 \%$ of the students thought their HVC education was inadequate, and about $83 \%$ noticed unnecessary testing. Our study findings reaffirm those of a single-site study in which $93 \%$ of students noted unnecessary testing. ${ }^{?}$

In this study, many students perceived HVC education as inadequate and reported wanting HVC principles added to their training and an HVC curriculum incorporated into 
TABLE. Student Observations of Behavior That May Hinder Practice of High-Value Care ${ }^{a}$

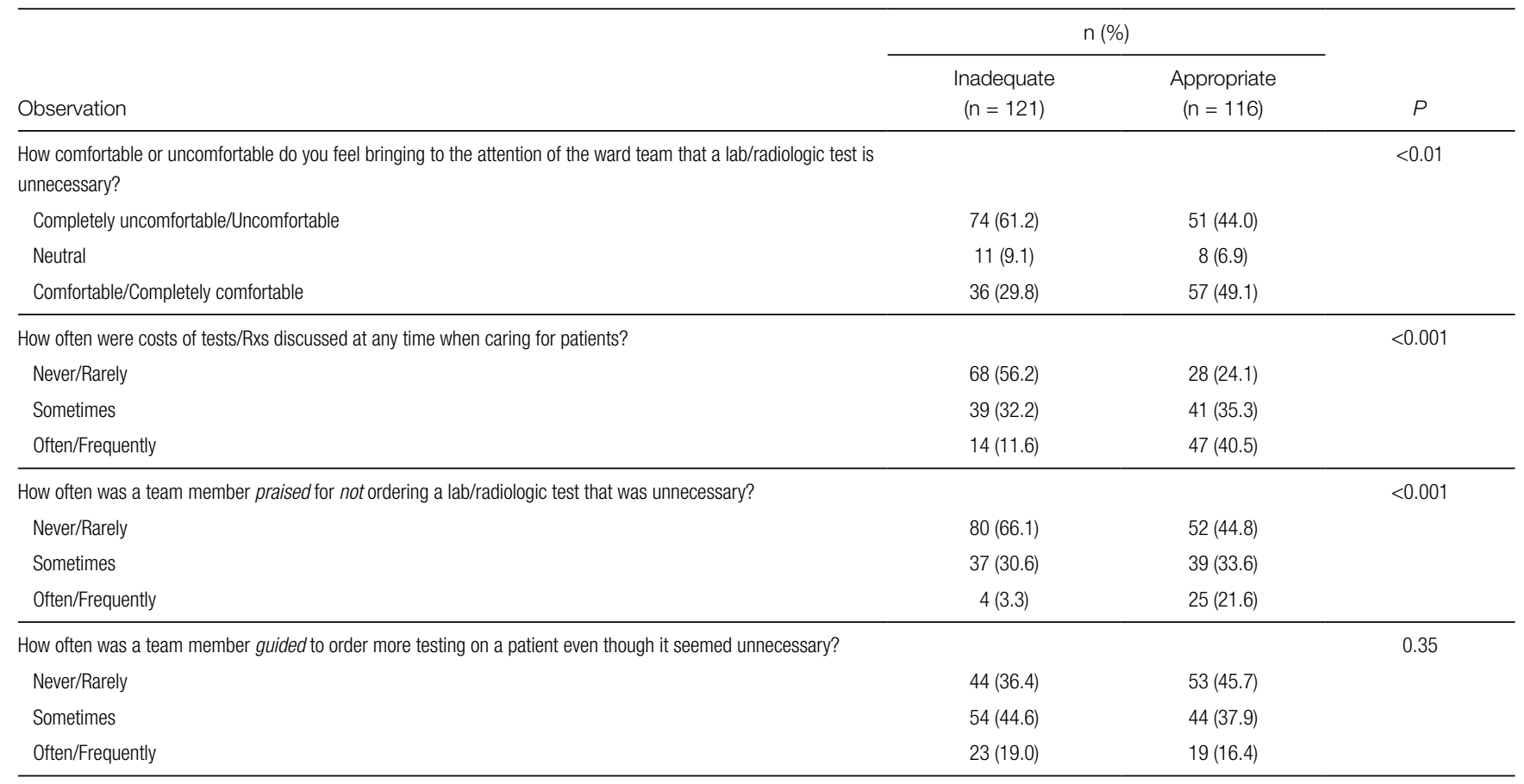

aResponses of students who reported that education in high-value cost-conscious care was inadequate or appropriate.

daily rounds. Students who perceived HVC education as inadequate were significantly less comfortable bringing an unnecessary test to the attention of the ward team and noticed less discussion about costs and less praise for avoiding unnecessary tests. One institution had a significantly higher proportion of students perceiving their HVC education as adequate and noticing more discussions about test costs. This institution was the only one that incorporated discussions about test costs into its curriculum during the study periodwhich may account for its students' perceptions.

This study had a few limitations. First, as the survey was administered after the IM clerkships, students' responses may have been subject to recall bias. We minimized this bias by allowing 1 week for survey completion. Second, given the $53 \%$ response rate, there may have been response bias. However, one institution's demographics showed no significant differences between responders and nonresponders with respect to age, sex, ethnicity, or type of degree. Third, students' understanding of what tests and treatments are necessary and unnecessary may be relatively underdeveloped, given their training level. One study found that medical students with minimal clinical experience were able to identify unnecessary tests and treatments, but this study has not been validated at other institutions. ${ }^{\text {? }}$

Efforts to increase HVC education and practice have focused on residents and attendings, but our study findings reaffirm that HVC training is much needed and wanted in undergraduate medical education. Studies are needed to test the effectiveness of HVC curricula in medical school and the ability of these curricula to give students the tools they need to practice HVC.

Disclosures: Dr. Pahwa received support from the Johns Hopkins Hospitalist Scholars Fund, and Dr. Cayea is supported by the Daniel and Jeanette Hendin Schapiro Geriatric Medical Education Center. The sponsors had no role in study design, methods, subject recruitment, data collection, data analysis, or manuscript preparation. The authors have no conflicts of interest to disclose.

\section{References}

1. Clerkship Directors in Internal Medicine, Society of General Internal Medicine. CDIM-SGIM Core Medicine Clerkship Curriculum Guide: A Resource for Teachers and Learners. Version 3.0. http://connect.im.org/p/cm/ld/fid=385. Published 2006. Accessed May 12, 2015.

2. Patel MS, Reed DA, Smith C, Arora VM. Role-modeling cost-conscious care-a national evaluation of perceptions of faculty at teaching hospitals in the United States. J Gen Intern Med. 2015;30(9):1294-1298.

3. Tek Sehgal R, Gorman P. Internal medicine physicians' knowledge of health care charges. J Grad Med Educ. 2011;3(2):182-187.

4. Patel MS, Reed DA, Loertscher L, McDonald FS, Arora VM. Teaching residents to provide cost-conscious care: a national survey of residency program directors. JAMA Intern Med. 2014;174(3):470-472.

5. Graham JD, Potyk D, Raimi E. Hospitalists' awareness of patient charges associated with inpatient care. J Hosp Med. 2010;5(5):295-297.

6. Detsky AS, Verma AA. A new model for medical education: celebrating restraint. JAMA. 2012;308(13):1329-1330.

7. Tartaglia KM, Kman N, Ledford C. Medical student perceptions of cost-conscious care in an internal medicine clerkship: a thematic analysis. J Gen Intern Med. 2015;30(10):1491-1496. 